\title{
Re-analysis of 201 I Liberia Malaria Indicator Survey data to provide project- level IPTp, ITN and malaria treatment estimates: Rebuilding Basic Health Services Project
}

\author{
Deirdre Rogers ${ }^{1 *}$ and Bal Ram Bhui ${ }^{2}$ \\ ${ }^{1} \mathrm{JSI}$ Research and Training Institute, Inc. 44 Farnsworth Street, Boston, MA 02210, USA \\ ${ }^{2}$ Rebuilding Basic Health Services Project, JSI/Liberia
}

\begin{abstract}
Malaria is one of many components addressed by the United States Agency for International Development (USAID) funded Rebuilding Basic Health Services (RBHS) project. According to the 20II Liberia Malaria Indicator Survey (MIS) report, 50\% of pregnant women received the recommended two doses of intermittent preventive treatment (IPTP) for malaria during their last pregnancy, though project records were showing coverage of $80 \%$. The discrepancy led USAID to question the project, which in turn led to a reanalysis of 20II MIS data. Despite limitations in generalizing the findings, the exercise proved to be a beneficial and costeffective evaluation method, and the analysis was expanded to include prevalence, diagnosis and prompt treatment of children with fever, and of mosquito net usage by children under 5 years of age and by pregnant women. This paper describes the methods, findings and the benefits and limitations in using secondary survey data to provide project-level coverage estimates.
\end{abstract}

Keywords: Malaria; Evaluation; Malaria Indicator Survey; Liberia; USAID

\section{Résumé}

Le paludisme est une des nombreuses composantes adressées par le United States Agency for International Development (USAID) a financé le projet Rebuilding Basic Health Services (RBHS). Selon le rapport d'enquête sur les indicateurs du paludisme Libéria 201 I (MIS), 50\% des femmes enceintes ont reçu les deux doses recommandées de traitement préventif intermittent (IPTP) du paludisme pendant leur dernière grossesse, bien que les dossiers des projets montraient une couverture de $80 \%$. L'écart a conduit USAID à remettre en question le projet, qui à son tour conduit à une nouvelle analyse des données de 201 I MIS.Malgré les limites à la généralisation des résultats, l'exercice s'est avéré être une méthode d'évaluation bénéfique et rentable, et l'analyse a été élargi pour inclure la prévalence, le diagnostic et le traitement rapide des enfants atteints de la fièvre et de l'utilisation des moustiquaires par les enfants de moins de 5 ans l'âge et les femmes enceintes. Cet article décrit les méthodes, les résultats aussi que les avantages et les limites des données d'enquête secondaires pour fournir des estimations de couverture au niveau du projet.

Mots clé: paludisme; evaluation; enquête sur les indicateurs du paludisme; Libéria; USAID

\footnotetext{
* Corresponding Author: Deirdre Rogers

Phone: +1-617-385-3895; Fax: +1-617-482-0617

drogers@jsi.com
} 


\section{Introduction}

The Rebuilding Basic Health Services (RBHS) project, funded by the United States Agency for International Development (USAID), is the United States government's major initiative in support of Liberia's Ministry of Health and Social Welfare (MOHSW). RBHS is a partnership led by JSI Research and Training Institute, Inc. (JSI), with partners Jhpiego, the Johns Hopkins University Center for Communication Programs, and Management Sciences for Health. The project runs from November 5, 2008 through October 31, 2014. Malaria is one of many components addressed in the project, with activities having been implemented in five out of Liberia's 15 counties. According to the 201 I Liberia Malaria Indicator Survey (MIS) report, only $50 \%$ of pregnant women received the recommended two doses of intermittent preventive treatment (IPTp) for malaria during their last pregnancy (among women who had a live birth in the last two years and where at least one dose was received during an antenatal care (ANC) visit). However, during the same period, the RBHS project was reporting coverage of $80 \%$ using administrative reports. The discrepancy led USAID to question the project, which in turn led to a reanalysis of 201 I MIS data, comparing MIS data collected in RBHS facility catchment areas to data collected in non-RBHS facility catchment areas to assess differences in IPT coverage.

The analysis was found to be highly informative to both USAID and the RBHS project. As a result, the analysis was expanded to include two other project/non-project analyses: prevalence, diagnosis and prompt treatment of children with fever; and mosquito net usage by children under 5 years of age and by pregnant women.

The process involved mapping the project target areas over those captured in the 20II MIS using Arc-GIS, identifying the appropriate comparison groups, and performing odd ratios and chi-square tests of independence using SAS. In order to compare to results reported in the 20I I Liberia MIS report, the same weights used in the MIS were applied to correct for the unequal probability of selection. However, since the 201 I Liberia MIS was designed using cluster sampling to generate robust estimates at the regional and national levels, there are limitations in generalizing the findings to the RBHS project level. Nonetheless, the exercise proved to be a beneficial and cost-effective tool contributing to other project evaluation activities.
This paper describes the process of designing and conducting the analysis, and the benefits and limitations in using secondary survey data to provide project-level coverage estimates.

\section{Literature Review and Theoretical \\ Framework}

The MEASURE DHS website

(http://www.measuredhs.com) maintains a list of Further Analysis publications that consist of reanalysis studies of Demographic Health Survey (DHS) data from single countries in order to further elucidate key program and policy issues; similar to the intended purpose of our reanalysis. We reviewed each of the 69 English language Further Analysis documents to assess common analytical practices for studies that either: (I) used the DHS data to conduct analyses at levels other than those for which the data was sampled, or (2) presented unweighted findings for analyses looking at association between variables (as opposed to calculations of incidence, prevalence, means or rates). We were unable to find examples of studies reanalyzing DHS data at levels other than at the national and/or regional levels for which the data was originally sampled, and thus were not able to identify 'best practices' in terms of describing the limitations of interpretation when conducting an analysis such as ours to generate project-specific estimates. However we did find three studies that presented unweighted data for multivariate analyses with the justification that it preserved the "one respondent/one response" relationship. ${ }^{\text {ii iii }}$ We subsequently consulted a senior biostatistician at Boston University, iv and decided that it made the interpretation easiest to uniformly apply the standard weights used in the 20II MIS, and to present prevalence data in the same table format as the 20II MIS report.

\section{Data and Methods}

Odds ratios were calculated and a chi-square test of independence was performed to compare the RBHS project areas to non-project areas for each of the following: (I) the probability of appropriately receiving two doses of IPT among women who had a live birth in the last two years, at least one dose provided during an ANC visit; (2) percent of children under five years of age and pregnant women who slept under an insecticide treated net the night before the survey; and, (3) children under five with a fever receiving antimalarial drugs on the same or next day. We employed a 0.05 significance level for all tests. DHS weights were consistently 
applied. SAS was used for all analyses using PROC FREQ.

\section{Determining RBHS Project Clusters}

To determine which MIS clusters were in RBHS facility catchment areas, we obtained the 201I cluster listing from the National Malaria Control Program, and mapped the clusters using Arc-GIS. The clusters were reviewed and confirmed as project areas by RBHS project county coordinators. At the time of this analysis, the 20II MIS cluster GPS coordinates were not yet available through MEASURE DHS, however it was confirmed that the coordinates were approximately the same as the previous 2009 Liberia MIS. There were a total of I50 clusters nationwide included in the 20 I I MIS. Out of that there were $4 \mathrm{I}$ clusters in five RBHS supported counties (Bong, Nimba, Lofa, Grand Cape Mount and River Gee). Out of the 4 I clusters, 26 clusters were located within catchment areas of RBHS-supported health facilities.
Identifying the Appropriate Comparison Groups

We first compared RBHS clusters in the five counties (Bong, Lofa, Nimba, River Gee and Grand Cape Mount) ( $n=26$ clusters) to all non-RBHS clusters (across the 15 counties) ( $n=124$ clusters), and then compared RBHS clusters in the five counties ( $n=26$ clusters) to non-RBHS clusters in the five counties $(n=16)$. The latter resulted in insufficiently powered sample sizes, and thus we compared project counties to all non-project counties for all conducted analyses.

Calculating the IPTP Denominator: Births less than two years

To match the analysis in the 20II MIS report, we calculated the denominator of women with a live birth in the last two years using the DHS Century Month Code variables for child birth and date of interview (as recommended by DHS).

Table I. Prophylactic use of antimalarial drugs and use of intermittent preventive treatment (IPTp) by women during pregnancy

Percentage of women age 15-49 with a live birth in the two years preceding the survey who, during the pregnancy preceding the last birth, took any antimalarial drug for prevention, who took one dose of SP/Fansidar, and who received IPTp, by RBHS project area, Liberia 201 I

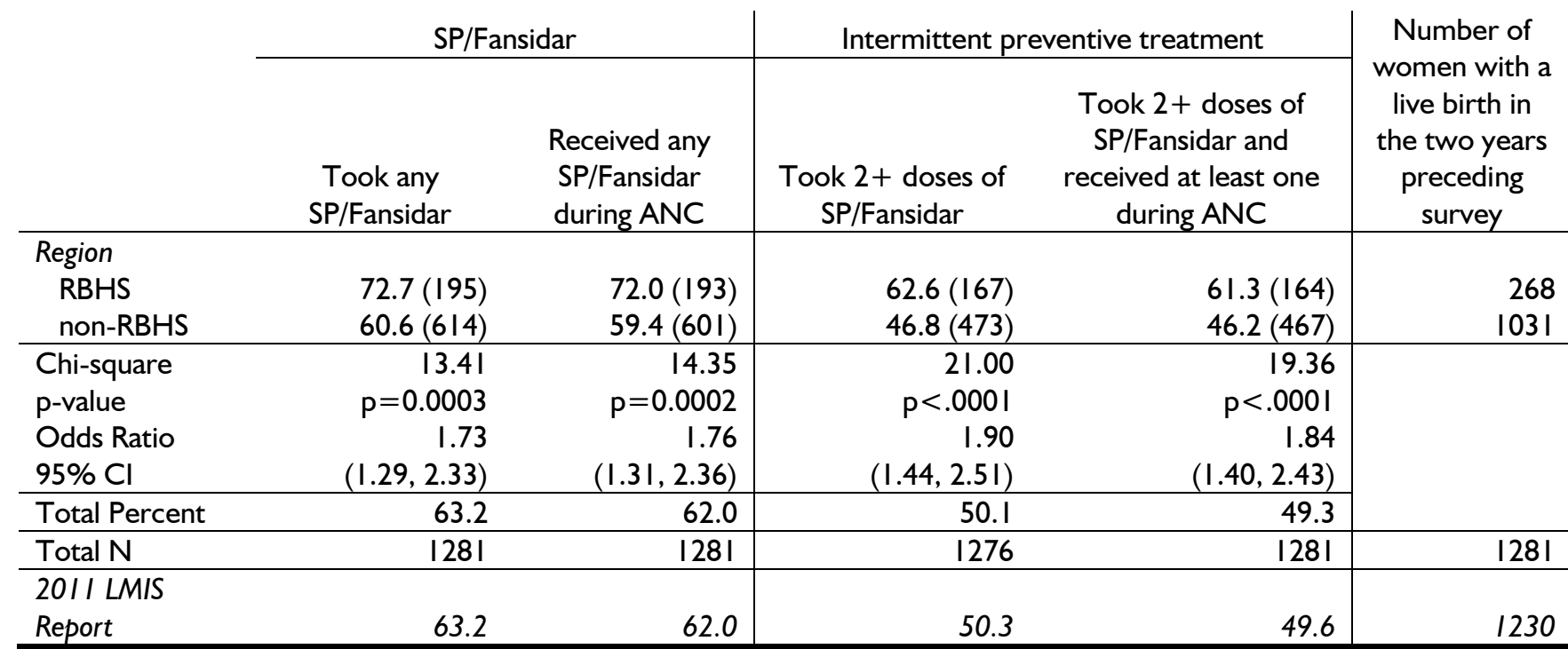

\section{Results}

Intermittent Preventive Treatment of Pregnant Women (IPTp)

Table I shows IPTp re-analysis findings according to RBHS project/non-project, compared to official MIS 20 I I report data.

Eligible women in RBHS areas (61\%) are significantly $(p<.000 \mathrm{I})$ more likely to have appropriately received IPT2 than in non-RBHS areas (46\%). The odds for having appropriately received IPT2 in the project catchment area was I.84 times the odds in the non-project catchment area $(95 \% \mathrm{Cl}=\mathrm{I} .40$, 2.43).

Liberia MIS clusters in RBHS facility catchment areas consistently show better coverage in each component indicator comprising the composite Intermittent Preventive Treatment indicator. Each difference was at least twelve percentage points higher in RBHS clusters. All differences were statistically significant as can be seen in Table I.

We also looked at RBHS administrative reports on IPTp coverage. To be comparable with the survey period, we took the average IPTp coverage 
reported for past two years January 2010 to December 2011 , which was $73 \%$, compared to $61 \%$ as shown in Table I using MIS data. Some possible explanations for the lower estimate obtained through the 201I MIS are due to limitations of self-report and recall bias, or overreporting of project health facilities. Furthermore, the 201 I MIS was only conducted in $80 \%$ of RBHS facility catchment areas, and thus the reanalyzed MIS data may not have been truly representative of the project area overall.

Use of mosquito nets by children under 5 years of age and pregnant women

Table 2 shows re-analysis findings of mosquito net usage by children under five years of age and by pregnant women in RBHS project and non-project areas, and overall compared to official MIS 20II report data.

The Liberia MIS clusters in RBHS facility catchment areas consistently show better coverage for both children under five years of age and for pregnant woman except for children who slept under a net among households with at least one insecticidetreated net (ITN) (64\% project vs. $67 \%$ nonproject). None of the differences, however, were statistically significant. It is likely, especially with the pregnant woman sample, that the sample sizes were simply too small to be able to provide robust estimates. 
Table 2. Use of mosquito nets by children under 5 years of age and pregnant women, RBHS vs. non-RBHS clusters

Percentage of the de facto household population who slept the night before the survey under a mosquito net (treated or untreated), under an insecticide-treated net (ITN), under a long-lasting insecticidal net (LLIN), and under an ITN or in a dwelling in which the interior walls have been sprayed against mosquitoes (IRS) in the past I2 months; and among the de facto household population in households with at least one ITN, the percentage who slept under an ITN the night before the survey, by RBHS and non RBHS areas, Liberia 2011

\begin{tabular}{|c|c|c|c|c|c|}
\hline & \multicolumn{4}{|c|}{ Household Population } & $\begin{array}{c}\text { Household population in } \\
\text { households with at least one ITN' }\end{array}$ \\
\hline & $\begin{array}{l}\text { Percentage who } \\
\text { slept under any net } \\
\text { last night }\end{array}$ & $\begin{array}{l}\text { Percentage who } \\
\text { slept under an ITN' } \\
\text { last night }\end{array}$ & $\begin{array}{c}\text { Percentage who slept } \\
\text { under an LLIN last } \\
\text { night }\end{array}$ & $\begin{array}{l}\text { Percentage who slept under an ITN' last } \\
\text { night or in dwelling sprayed with IRS } \\
\text { past } 12 \text { months }\end{array}$ & $\begin{array}{c}\text { Percentage who slept under an ITN' } \\
\text { last night }\end{array}$ \\
\hline \multicolumn{6}{|c|}{ Children Under 5} \\
\hline \multicolumn{6}{|l|}{ Region } \\
\hline RBHS & $40.9(282)$ & $40.0(276)$ & $39.8(274)$ & $43.8(302)$ & $64.0(276)$ \\
\hline non-RBHS & $37.4(990)$ & $36.3(963)$ & $36.0(953)$ & $42.6(1129)$ & $67.2(963)$ \\
\hline Chi-square & 2.85 & 3.08 & 3.38 & 0.34 & 1.53 \\
\hline p-value & $p=0.0917$ & $p=0.0794$ & $p=0.0662$ & $p=0.5612$ & $p=0.2157$ \\
\hline Odds Ratio & 1.16 & 1.17 & 1.17 & 1.05 & 0.87 \\
\hline $95 \% \mathrm{Cl}$ & $(0.98,1.37)$ & $(0.98,1.38)$ & $(0.99,1.40)$ & $(0.89,1.24)$ & $(0.69,1.09)$ \\
\hline Total Percent & 38.1 & 37.1 & 36.7 & 42.9 & 66.5 \\
\hline Total N & 3340 & 3340 & 3340 & 3340 & 1864 \\
\hline $201 /$ MIS Report & 38.1 & 37.1 & 36.7 & 42.9 & 68.0 \\
\hline Total N & 3352 & 3352 & 3352 & 3352 & 1827 \\
\hline \multicolumn{6}{|c|}{ Pregnant Women } \\
\hline RBHS & $47.9(35)$ & $46.5(34)$ & $46.5(34)$ & $46.5(34)$ & $80.6(34)$ \\
\hline non-RBHS & $38.3(111)$ & $37.2(108)$ & $37.2(108)$ & $45.1(131)$ & $74.2(108)$ \\
\hline Chi-square & 2.26 & 2.14 & 2.14 & 0.05 & 0.71 \\
\hline $\mathrm{p}$-value & $p=0.1329$ & $p=0.1439$ & $p=0.1439$ & $p=0.8318$ & $p=0.4004$ \\
\hline Odds Ratio & 1.45 & 1.47 & 1.47 & 1.06 & 1.44 \\
\hline $95 \% \mathrm{Cl}$ & $(0.86,2.50)$ & $(0.88,2.47)$ & $(0.88,2.47)$ & $(0.63,1.77)$ & $(0.61,3.36)$ \\
\hline Total Percent & 40.2 & 39.0 & 39.0 & 45.4 & 75.7 \\
\hline Total N & 363 & 363 & 363 & 363 & 187 \\
\hline $201 /$ MIS Report & 40.2 & 39.0 & 39.0 & 45.4 & 77.4 \\
\hline Total N & 363 & 363 & 363 & 363 & 183 \\
\hline
\end{tabular}

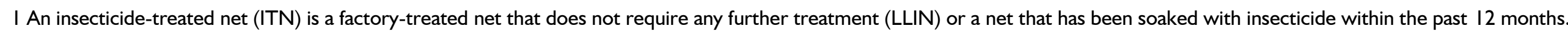

2 Indoor residual spraying (IRS) is limited to spraying conducted by a government, private or non-governmental organization. 


\section{Table 3. Prevalence, diagnosis, and prompt treatment of children with fever}

Percentage of children under age five with fever in the two weeks preceding the survey; and among children under age five with fever, the percentage for whom advice or treatment was sought from a health facility, provider, or pharmacy, the percentage who had blood taken from a finger or heel, the percentage who took artemisinin-based combination therapy (ACT), the percentage who took ACT the same or next day following the onset of fever, the percentage who took antimalarial drugs, and the percentage who took the drugs the same or next day following the onset of fever, by RBHS and non RBHS areas, Liberia 20II

Among children U5

Percentage with fever in the two weeks preceding the survey

\begin{tabular}{|c|c|c|c|c|c|c|c|}
\hline & $\begin{array}{l}\text { Percentage with fever in the two } \\
\text { weeks preceding the survey }\end{array}$ & $\begin{array}{l}\text { Percentage for whom } \\
\text { advice or treatment was } \\
\text { sought from a health facility, } \\
\text { provider or pharmacy }{ }^{\prime}\end{array}$ & $\begin{array}{l}\text { Percentage who had } \\
\text { blood taken from a } \\
\text { finger or heel for } \\
\text { testing }\end{array}$ & $\begin{array}{c}\text { Percentage } \\
\text { who took } \\
\text { ACT }\end{array}$ & $\begin{array}{l}\text { Percentage who } \\
\text { took ACT same } \\
\text { or next day }\end{array}$ & $\begin{array}{l}\text { Percentage } \\
\text { who took } \\
\text { antimalarial } \\
\text { drugs }\end{array}$ & $\begin{array}{c}\text { Percentage who } \\
\text { took antimalarial } \\
\text { drugs same or } \\
\text { next day }\end{array}$ \\
\hline \multicolumn{8}{|l|}{ Region } \\
\hline RBHS & 48.9 (309) & $64.2(181)$ & $34.5(97)$ & $44.4(125)$ & $27.2(76)$ & $60.8(171)$ & $37.2(104)$ \\
\hline Non-RBHS & $46.1(1107)$ & $57.6(566)$ & $33.1(326)$ & $40.1(395)$ & $25.3(249)$ & $58.1(57 I)$ & $35.9(353)$ \\
\hline Chi-square & 1.60 & 3.97 & 0.19 & 1.62 & 0.41 & 0.65 & 0.16 \\
\hline Odds Ratio & 1.12 & 1.32 & 1.06 & 1.19 & 1.10 & 1.12 & 1.06 \\
\hline $95 \% \mathrm{Cl}$ & $(0.94,1.33)$ & $(1.00,1.74)$ & $(0.8 I, I .4 I)$ & $(0.91,1.55)$ & $(0.82,1.49)$ & $(0.85,1.47)$ & $(0.81,1.39)$ \\
\hline Total Percent & 46.7 & 59.7 & 33.5 & 41.1 & 25.7 & 58.7 & 36.2 \\
\hline Total N & $3034^{2}$ & 1264 & 1264 & 1264 & 1264 & 1264 & 1264 \\
\hline 20II LMIS Report & 49.2 & 59.7 & 33.3 & 39.7 & 24.5 & 57.1 & 35.0 \\
\hline Total N & 2876 & 1416 & 1416 & 1416 & 1416 & 1416 & 1416 \\
\hline
\end{tabular}

Among children U5 with fever

2 We were not able to replicate the analysis to come up with the same denominators as in the 20II MIS report 
Prevalence, diagnosis, and prompt treatment of children with fever

Table 3 shows re-analysis findings of the prevalence of fever in children under five years of age, as well as treatment seeking, diagnosis, treatment and the timing of treatment, comparing RBHS project vs. non-project areas, and overall compared to official MIS 20I I report data.

Data shows those in RBHS project areas consistently with higher coverage across all indicators: proportion of children under five years of age with a fever for whom treatment was sought, who took ACT, who took ACT in a timely fashion, who took any antimalarial drug, and who took any antimalarial drug in a timely fashion. However, none of the differences were statistically significant.

We also looked at RBHS project administrative reports on ACT coverage among children under five years of age presenting with fever, showing very high coverage of $90 \%$. As Table 3 shows, the selfreported ACT coverage from the MIS among children with fever in RBHS areas is only $44 \%$. The difference can at least in part be explained by the fact that the Liberia MIS (demand side) asked for ACT coverage only among children with a fever in the past two weeks, whereas the RBHS facility records (supply side) include all ACT provided to any child during the reporting period presenting with a fever. Further, the MIS does not limit the indicator to those for whom treatment was sought in a public sector facility.' Also, children with a fever were borderline significantly more likely to be brought for treatment in RBHS project areas than non-RBHS areas (64\% vs. $58 \%, p=0.05)$. Thus one would expect higher ACT coverage from facility records in RBHS areas than non-RBHS areas. Furthermore, the 20II MIS was not conducted in one-fifth of RBHS facility catchment areas. Finally, there may be limitations of self-report and recall bias in any survey, and/or over-reporting on the part of the project facilities.

\section{Discussion}

The 20 I I Liberia MIS was administered using cluster sampling to generate robust estimates at the regional and national levels. Clusters were weighted

\footnotetext{
${ }^{1}$ The MIS ACT indicator includes treatment sought from: government hospitals, government health centers, government health posts, government mobile clinic, government community health workers, other public sector sources, private hospitals/clinics, private pharmacies, private doctors, private mobile clinics, private community health workers, and other private sector. It excludes treatment sought in shops, markets or through traditional practitioners.
}

according to their respective surrounding community population sizes to allow for regional and national estimates. Since we were not interested in extrapolating to the entire country, but simply comparing the RBHS project areas included in the survey to the non-RBHS areas included in the survey, the raw, unweighted data was initially used for multivariate analyses, and which tended to show greater differences and statistical significance between project and non-project areas. Following a review of the Further Analysis publications on the MEASURE DHS website to assess how this has been handled in the past, we decided that it was best to consistently apply DHS weights since they are calculated not only based on population size, but also to account for intentional over- and undersampling in the sample design, and for large discrepancies in response rate from one area to the next. Furthermore, we opted to parallel, to the extent possible, the MIS analysis and present prevalence estimates in the same format and layout to increase ease of interpretation. Nonetheless, since the MIS data was not originally collected for RBHS project purposes (but rather to make regional and national estimations), we were careful to review the findings with the understanding that they cannot be generalized to the larger populations from which they were drawn (including to the larger RBHS project catchment area). Still, conclusions about the project and non-project differences among this sample provided beneficial project management information.

\section{Conclusion}

Intermittent Preventive Treatment of Pregnant Women RBHS project catchment areas (61\%) did significantly better than non-RBHS (46\%) in the 201 I MIS survey $(p<.0001)$. However, the RBHS project reported figure from project records for the comparable period $(80 \%)$ was much higher than the MIS estimate $(61 \%)$. There are a number of potential explanations for the discrepancy including over-reporting by RBHS, limitations of self-reporting and recall bias in the MIS, and nonrepresentativeness of the RBHS clusters in the MIS data.

Use of mosquito nets by children under five years of age and pregnant women

Though the RBHS project catchment areas generally show better coverage for both children under five years of age and for pregnant woman, none of the differences were statistically significant. This analysis suffered from an inadequately powered sample size, 
in particular among the analysis for pregnant woman.

Prevalence, diagnosis, and prompt treatment of children with fever

In general, eligible under-five children with a fever in RBHS project areas are more likely to receive timely treatment for fever than in non-RBHS areas. The MIS reanalysis shows that RBHS project area clusters did better than non-RBHS clusters across all indicators: proportion of children under five with a fever for whom treatment was sought, who took $A C T$, who took ACT in a timely fashion, who took any antimalarial drug, and who took any antimalarial drug in a timely fashion. However, none of the differences were statistically significant. Further, the 201 I MIS survey found overall $40 \%$ coverage of ACT among children with a fever in the two weeks prior to the survey, whereas the RBHS reported figure from project records for the comparable period was $90 \%$. One contributing factor may be the fact that those in RBHS areas were borderline significantly more likely to seek treatment than those in non-RBHS areas (64\% vs. $58 \%, p=0.05)$. There are a number of potential other explanations, such as over-reporting by RBHS, limitations of selfreporting and recall bias by MIS, and nonrepresentativeness of the RBHS clusters in the MIS data.

We found working with the MIS dataset challenging in recreating the calculations performed by MIS analysts in order to come up with exactly the same denominators. For example, in Table I, the 201 I MIS reported a total of 1,230 births in the last 2 years; 5 I fewer than our I,28I. Similar discrepancies can be seen in Tables 2 and 3. We were unable to determine the reason for these discrepancies despite correspondence with MEASURE DHS analysts, but concluded that for our primary purpose of comparing project to non-project areas, it was inconsequential.

The DHS reanalysis allowed us to compare project indicators collected in the survey to those also collected through project records, thus helping validate the accuracy of the project tools, as well as provide a rough assessment of the impact of project activities at the household level. In this sense, it was a beneficial exercise.

On the other hand, challenges in conducting the analysis in terms of being able to specifically recreate the MIS variable/indicator definitions, inadequate sample sizes for some of the sub population analysis, and the larger issue of generalizability to the project area led us to question whether it would equally be a good use of project staff time on future projects.

The standardized and rigorous implementation of the Demographic and Health Surveys provides a wealth of largely unharnessed data for project management and evaluation purposes. However, to maximize cost effectiveness, it is suggested that health projects-and in particular those that are specifically aligned with national priorities-partner with DHS to oversample the clusters from project areas to provide a more robust assessment of the effectiveness of project activities at the household level. Even a single DHS during the second half of a project timeline would be beneficial in contributing to project management and overall project evaluation.

\section{Authors' Contribution}

Deirdre Rogers, MS, JSI Research and Training Institute, Inc. 44 Farnsworth Street, Boston, MA 02210, USA was responsible for the literature search, figures, study design, data analysis, data interpretation and writing.

Bal Ram Bhui, MPH, MBA, Rebuilding Basic Health Services Project, JSI/Liberia, reviewed the draft and provided ideas on interpretation of findings.

\section{References and Acknowledgement}

'Bizuneh, Genene, Solomon Shiferaw, and Yilma Melkamu. 2008. Unmet Need and Evaluation of Programme Options to Meet Unmet Need for Contraception in Ethiopia, 2000-2005: Further Analysis of the 2000 and 2005 Ethiopia Demographic and Health Surveys. Calverton, Maryland, USA: Macro International Inc.

ii Mishra, Vinod, Rathavuth Hong, Pav Govindasamy, and Livia Montana. 2008. Evaluating HIV Seroprevalence Estimates from Ethiopia: Further Analysis of the 2005 Ethiopia Demographic and Health Survey. Calverton, Maryland, USA: Macro International Inc.

iii Korra, Antenane. 2002. Attitudes toward Family Planning, and Reasons for Nonuse among Women with Unmet Need for Family Planning in Ethiopia. Calverton, Maryland USA: ORC Macro.

iv Dr. Tim Heeren, PhD, Boston University, Department of Biostatistics, 801 Massachusetts Ave, Boston, MA USA, provided valuable statistical advice on the use and interpretation of unweighted data. 